Episteme, I7, I (2020) I-27 (C) Cambridge University Press 20I8. This is an Open Access article, distributed under the terms of the Creative Commons Attribution-NonCommercial-NoDerivatives licence (http://creative commons.org/licenses/by-nc-nd/4.o/), which permits non-commercial re-use, distribution, and reproduction in any medium, provided the original work is unaltered and is properly cited. The written permission of Cambridge University Press must be obtained for commercial re-use or in order to create a derivative work.

doi:Io.IOI7/epi.20I8.9

\title{
DELIBERATIONALLY USELESS CONDITIONALS
}

\section{KAROLINA KRZYŻANOWSKA}

k.h.krzyzanowska@uva.nl

\begin{abstract}
Decision theorists tend to treat indicative conditionals with reservation, because they can easily lead a deliberating agent astray. However, many indicatives can be very helpful in contexts of deliberation, so denying them all a role in such contexts seems to be overkill. We show that a recently revived inferential view on conditionals provides a straightforward explanation of why some indicatives are unassertable in contexts of deliberation and hints at a way of telling "deliberationally useless" and "deliberationally useful" conditionals apart.
\end{abstract}

We make choices all the time. We may decide to leave earlier for work if we want to avoid traffic jams, to buy fruit instead of cookies for a healthy diet, or to learn French to improve our chances on the job market. We constantly deliberate over possible consequences of actions we might or might not undertake. Some of the decisions we make every day are made on the spot, without any deliberation, any reasoning at all, but others are carefully thought through. Whenever conscious decision making takes place, people are very likely to entertain conditionals like:

(I) a. If I add some fresh herbs, the sauce will be tastier.

b. If we take a cab, we won't be late for the concert.

c. If I accept this job, I will need to move to London.

Naturally, the process of deliberation does not always have to be a single agent affair. Other people may participate and advise a deliberating agent directly (2a) or indirectly $(2 b, 2 c)$ :

(2) a. If you add some fresh herbs, the sauce will be tastier.

b. If they take a cab, they won't be late for the concert.

c. If she accepts this job, she will need to move to London.

Yet it is a widespread assumption that conditionals of deliberation - that is, those conditionals that can guide a deliberating agent in their decision making - are counterfactuals or, to be more precise, future directed subjunctive conditionals of the form "If $\varphi$ were the case, then $\psi$ would happen.” Some researchers, for instance Allan Gibbard and William Harper, state this assumption as a matter of course:

In the first place, rational decision-making involves conditional propositions: when a person weighs a major decision, it is rational for him to ask, for each act he considers, what would happen 
if he performed that act. It is rational, then, for him to consider propositions of the form 'If I were to do $A$, then $C$ would happen.' (Gibbard and Harper 1978 : I 53)

Others, like James Joyce, point out that the goal of decision theory is:

to provide a rigorous formal analysis of the idea that a rational decision maker should evaluate her potential actions solely on the basis of their ability to cause desirable outcomes. (Joyce I999: I6I)

Consequently, they advocate for causal decision theory.

Since causal decision theorists believe that "causation is best understood in subjunctive terms" (Joyce I999: I7I), on this account the thoughts that deliberating agents are entertaining should not be phrased as indicatives, like in the examples (I) or (2). Instead, the relevant conditionals should be in the subjunctive mood:

(3) a. If you were to add some fresh herbs, the sauce would be tastier.

b. If they were to take a cab, they wouldn't be late for the concert.

c. If I were to accept this job, I would need to move to London.

Clearly, realising that whatever is expressed by the above conditionals' antecedents can bring about the state of affairs expressed by their consequents may help a deliberating agent to come to the right decision. Subjunctive conditionals like the ones above are particularly efficacious in expressing such relations between actions and states of affairs. It is not obvious, however, that the power to convey the relations that are of interest to a deliberating agent is unique to subjunctives: examples (Ia)-(Ic) and (2a)-(2c) seem to belie such a view. Moreover, subjunctive mood present in sentences $(3 a)-(3 c)$ does not guarantee at all that these conditionals share their acceptability conditions or their assertability conditions ${ }^{\mathrm{I}}$ with the paradigmatic cases of subjunctive counterfactual conditionals, such as $(4 \mathrm{~b})$ below (Adams 1970):

(4) a. If Oswald did not kill Kennedy, someone else did.

b. If Oswald had not killed Kennedy, someone else would have.

In fact, it has been argued that the subjunctive, “"were'd-up” future-directed conditionals that is, sentences of the form "If I were to do $\varphi, \psi$ would happen" - have the same assertability conditions as the corresponding, straightforward future directed conditionals, "If I do $\varphi, \psi$ will happen" (DeRose 2010). ${ }^{2}$ According to DeRose, both types share their assertability conditions with the paradigmatic indicative, namely, (4a). Consequently, DeRose views all conditionals of deliberation as semantically indicative.

It is not the goal of this paper to enter into the debate on the semantics of subjunctive future-directed conditionals, a matter about which we remain agnostic. But, since we

I Or their truth conditions if we believe that conditionals are truth-evaluable.

2 Throughout this paper, we take "subjunctive conditional" to be a grammatical notion, denoting conditional sentences in the subjunctive mood, regardless of their semantic or pragmatic features, while the term "counterfactual" will be used as a semantic notion. This distinction allows us to refer to controversial cases such as "were'd-up" conditionals in (3) as subjunctives without committing ourselves to any particular view on their semantics. 
second DeRose's view that straightforward indicatives ${ }^{3}$ such as those in the examples (I) or (2) can serve as conditionals of deliberation, we have to address some of the issues that DeRose's main thesis poses. More specifically, we are faced with a problem, well documented in the causal decision theory literature, that indicative conditionals can lead a deliberating agent astray. If we do not want to agree that all indicatives are potentially a threat to a deliberating agent, and, consequently, ban all of them from the contexts of deliberation, we need to find out what it is that makes a conditional a piece of bad advice.

In the following sections, I will define the notion of a conditional of deliberation, and discuss cases that appear to undermine the view that indicatives can be helpful in contexts of deliberation. Furthermore, I will show that what Keith DeRose identifies as the reason for a conditional to be a threat to a deliberating agent, namely, their dependence on backtracking grounds, is neither necessary nor sufficient for a conditional to turn out so. Finally, drawing from the linguistic and philosophical literature on so-called inferential conditionals, I will offer an alternative explanation of the phenomenon, providing a straightforward way to tell deliberationally useless and deliberationally useful conditionals apart.

\section{INDICATIVE CONDITIONALS OF DELIBERATION}

Before we discuss problems that indicative conditionals may pose when asserted in a context of deliberation, let us clarify the notions we are going to use throughout the paper. In particular, we need to clarify what it means for a conditional sentence to be a conditional of deliberation. First of all, it is important to emphasise that this term is not meant to denote a separate category of conditionals that would be distinct from any particular type of conditional sentences described by linguists such as Declerck and Reed (200I). Instead, a conditional of deliberation is a function that conditionals of many different types can assume.

In the opening sections, we stated that conditionals of deliberations are typically construed as those conditionals that can guide a deliberating agent in their decision making. However, not all conditionals that might be relevant and assertable in a context of deliberation should count as conditionals of deliberation. The term conditional of deliberation is meant to apply to only those conditional sentences that concern actions that a deliberating agent considers undertaking, on the one hand, and events or states of affairs that depend on those actions, on the other hand. Whenever an agent desires that a particular state of affairs comes about, a conditional of deliberation will suggest an action that the agent should take in order to achieve that goal. And when an agent is deciding between taking or not taking a particular action, a conditional of deliberation should provide them with information about consequences of this action.

More precisely, a conditional of deliberation can be defined in the following way:

Definition I. A sentence of the form "If $\varphi, \psi$ " 4 is a (simple) conditional of deliberation for an agent $\mathrm{A}$ in a context $\mathrm{C}$ if and only if $\psi$ expresses a proposition whose truth value is

3 Henceforth, indicatives or indicative conditionals simpliciter.

4 Limiting the class of conditionals to sentences with this particular surface structure is, of course, a simplification. Many sentences that do not even involve an if-clause, such as "Touch me and I will scream," are classified as indicative conditionals, while some "If $\varphi, \psi$ "-sentences are not. For more on this matter, see, e.g., Elder and Jaszczolt (2016). 
potentially of interest to $\mathrm{A}$, and $\varphi$ expresses a proposition whose truth value $\mathrm{A}$ can, in context $\mathrm{C}$, directly influence.

If $A$, in a context $C$, accepts "If $\varphi, \psi$," and desires $\psi$ to be the case, it would be rational for $A$ in $C$ to (attempt to) make $\varphi$ true. This can only be the case when $A$ is justified in accepting the conditional, and, when the conditional is itself deliberationally useful. Falling under the definition of a conditional of deliberation does not guarantee that given conditional is a piece of good advice.

It is important to note that a conditional of deliberation does not have to be explicitly about an action. However, propositions whose truth values an agent can directly influence will typically concern actions that the agent can take or immediate results of such actions. To illustrate the distinction between actions and their immediate results, let us consider the following pair of sentences:

(5) a. If you do not water this plant, it will die.

b. If this plant is not watered, it will die.

The antecedent of $(5 \mathrm{a})$ is clearly about an action, (an action of watering the plant), while ( 5 b), being in passive voice, does not seem to be. Grammatical differences notwithstanding, the two conditionals can be asserted by the same speaker in exactly the same circumstances. For instance, imagine a context in which Daisy, a deliberating agent, is a proud owner of a plant in a pot. She is also an absent-minded thirteen-year-old with a predisposition towards negligence. In this context, ( 5 a) asserted by Daisy's mother and addressed directly to Daisy is a clear case of a conditional of deliberation. Daisy has no reason not to accept the conditional, given that it is coming from a reliable source, namely, her mother, and she does not want its consequent to become true, that is, she does not want her plant to die. The conditional $(5 \mathrm{a})$ then informs Daisy that to prevent the plant from dying she needs to make sure that the antecedent of that conditional is not true. In other words, she must water the plant.

Although (5b) does not explicitly concern any actions, it is as good a conditional of deliberation for Daisy as (5a). However, assuming again that Daisy accepts the conditional and that she does not want its consequent, "the plant will die," to become true, (5a) tells her what she needs to do. By contrast, (5b) only informs her about what should be done, not by whom. In this sense, conditionals like ( $5 \mathrm{~b}$ ) concern actions implicitly.

Saying that $(5 \mathrm{a})$ and $(5 \mathrm{~b})$ can both function as conditionals of deliberation relative to the same agent in the same context is not to say the two sentences are equivalent (not even that they are equivalent qua conditionals of deliberation). The difference between the two types of conditionals is not so subtle after all: in the first case, Daisy is advised to water the plant herself, while, to follow the advice of the second conditional, she only needs to ensure that the plant is watered. She might, for instance, make a deal with her little brother, who would gladly water the plant for a bowl of ice cream, or she might leave the pot in the garden on a rainy day. Nevertheless, both types of conditionals serve perfectly well as conditionals of deliberation. $5^{5}$

5 Note that among the subjunctive, "“were'd-up" conditionals of deliberation, one can also find both implicit and explicit conditionals of deliberation, e.g.:

(6) a. If you weren't to water this plant, it would die.

b. If this plant were not to be watered, it would die. 
Our preliminary definition permits two types of conditionals to be classified as conditionals of deliberation: explicit conditionals targeted at particular agents and implicit conditionals that only indicate what needs to be done. There are, however, other types of conditionals that may be assertable in the contexts of deliberation, but are excluded by this definition. In particular, there is a class of conditionals which seem to be very closely related to the previously discussed cases of conditionals of deliberation such as (I), (3) or (5):

(7) a. If you want the sauce to taste better, you can add some fresh herbs.

b. If they don't want to be late for the concert, they should take a cab.

c. If I don't want to move to London, I shouldn't accept this job.

d. If you don't want this plant to die, you should water it.

Clearly, indicative conditionals falling under the schema "If an agent $A$ wants $\psi, A$ should $\varphi$ " can be useful in the context of decision making. Although they differ from the previously discussed examples grammatically, there is no reason to deny them the status of conditionals of deliberation. In fact, when asserted purposefully in contexts of deliberation, deontic conditionals like $(7 \mathrm{~d})$, can be thought of as paraphrases of the simple conditionals of deliberations such as $(5 \mathrm{a})$ that we discussed before. This is not to say that they do not differ in their meaning at all. What deontic conditionals do is that they make explicit the decision-theoretic relevance of their simple indicative counterparts. This is particularly evident in contexts in which a speaker and a deliberating agent are not the same person and in which a conditional of deliberation is meant as a piece of advice. In this kind of context, the speaker does not assert "If $\varphi, \psi$ " and wait for the agent to realise on their own that their desire for $\psi$ to be the case means that they should make $\phi$ happen. Rather, the speaker simply makes it immediately known to them. ${ }^{7}$ In our opinion, this is a good enough reason to revise the definition of a conditional of deliberation so that it encompasses both types: simple conditionals of deliberation defined in the Definition I and deontic conditionals:

Definition 2. A sentence of the form "If $\gamma, \xi$ " is a deontic conditional of deliberation for an agent $\mathrm{A}$ in a context $\mathrm{C}$ if and only if $\gamma$ is a propositional attitude report that attributes to $\mathrm{A}$ a desire that $\psi$ is true, and $\xi$ is a deontic statement that attributes to $\mathrm{A}$ an obligation to see to it that $\varphi$ is true. ${ }^{8}$

Definition 3. A sentence is a conditional of deliberation if and only if it is either a simple conditional of deliberation or it is a deontic conditional of deliberation.

It is not the purpose of this definition to capture all kinds of conditionals that a deliberating agent may find useful. Quite the contrary: we want the notion of a conditional of

6 It is perhaps worth noting that ( 5 b) asserted by Daisy's mother in the absence of Daisy, say, addressed to Daisy's father who does not care at all about the well-being of his daughter's plant, will not function as a conditional of deliberation, but as a mere statement of the relationships between states of affairs.

7 One could argue that, when they are asserted in contexts of deliberation, simple conditionals of deliberation pragmatically implicate their deontic relatives.

8 For the sake of simplicity, we leave out negative versions of deontic conditionals, such as "If you don't want $\psi, \ldots$, , assuming that these can be defined in terms of positive deontic conditionals. 
deliberation to have a relatively narrow scope, as it is conceivable that any conditional, be it indicative or subjunctive, may turn out to be assertable in some context of deliberation and even entertained by some deliberating agent. After all, our decisions are not always backed up by a single statement which would give us a simple recipe for solving the problem at hand. Many conditionals that a deliberating agent can consider before making a decision do not concern reasons to take or not to take particular actions. They may be used to express inferences, relationships between states of affairs, or even laws of nature. For instance, Daisy's mother, in the context discussed above, could tell her daughter "If the soil in the pot is this dry, you must have forgotten to water your plant again." Such a conditional is relevant to the issue Daisy and her mother are talking about, but it does not prompt the agent, Daisy, to undertake any particular action. It plays an important role in the mother's reasoning that leads her to realising that Daisy needs to be reminded about watering the plant and motivated to do it. Daisy may also assert, in this context, a conditional which would not classify as a conditional of deliberation. For instance, after hearing her mother's assertion, Daisy might make an association with what she learned in her biology class and say: "If the plant has no access to water, it cannot photosynthesise." Such a thought could affect how seriously Daisy takes her mother's advice, but it would still be ( $5 \mathrm{a}$ ) that would directly motivate her to take an action.

\section{THE PROBLEM OF BAD ADVICE}

Although the definition of a conditional of deliberation proposed in the previous section potentially excludes a great number of conditional sentences that could be useful for some agents in some contexts of deliberation, one could still argue that it is too broad. There are many indicatives that, in the light of this definition, classify as conditionals of deliberation, yet no competent language user would ever assert them as pieces of advice. These are conditional sentences that concern actions and states of affairs or events, and which might be taken by some agents as reasons to take or not to take these actions. Consider, for instance, the following sentences:

(8) a. If Charlie buys a Maserati, he will be able to afford staying in a 5-star hotel.

b. If Judy is so stressed, she is giving a talk soon.

Imagine Charlie, who needs to break it to his old friends that he cannot join them for a weekend in the Alps, a yearly tradition that they have been cultivating ever since they were in their twenties. The problem is that Charlie's friends like to stay in hotels way too expensive for his pocket. In the meantime, Bob, one of Charlie's wealthy friends spotted him at a Maserati dealership being courteously attended to by an employee. Unbeknownst to Bob, the employee is Charlie's prospective son-in-law. Bob does not know that Charlie's financial situation deteriorated over the last couple of years, though after seeing the expression on Charlie's face when they were discussing the details of their weekend trip, Bob began to suspect that Charlie might not be as well off as he used to be. Seeing Charlie in the Maserati dealership resolved Bob's doubts, however. He shared his observation with his friends and, finally, asserted (8a). Imagine now that Charlie overhears Bob's conditional. Charlie's buying a Maserati would be good evidence for his being rich and, consequently, being able to afford a weekend in a luxurious resort, which would be a highly desirable outcome for him. Yet, clearly, Charlie would never 
take (8a) to be a piece of advice suggesting a way for him to achieve the state of affairs expressed by the consequent.

Similarly, Judy might be a graduate student who tends to get incredibly stressed whenever she has a talk to give, so a good friend who recognises signs of high tension in Judy's behaviour is justified in asserting (8b). Incidentally, Judy believes that speaking at conferences or seminars, however taxing, is good for her academic career, so she would appreciate any tips on how to get more opportunities to present her work. Yet she would never mistake ( $8 \mathrm{~b})$ for a piece of advice, even if she agreed with what it says. Getting stressed on purpose, whatever it would amount to, is simply not a way of getting another opportunity to give a talk.

The indicative conditionals in (8), even though they are conditionals of deliberation, do not provide the deliberating agents with any valuable advice on what they should do, even though these conditionals' antecedents constitute good evidence for their consequents and the consequents express states of affairs highly desirable from the perspectives of the agents concerned. Fortunately, these two sentences would never be asserted as pieces of advice and they would never be mistaken as such. This is not always the case though: sometimes indicatives can appear to be useful tips when they really are not, and often it is not obvious how to distinguish a good piece of advice from an advice-like statement which could lead to a bad decision. Furthermore, the same sentence can serve as a good piece of advice for one agent in one context, but a detrimental one for another agent in another context. This is why we must not narrow the scope of the definition to exclude (8) and similar sentences from being classified as conditionals of deliberation. We must, however, emphasise, adopting terminology introduced by Keith DeRose (2010), that conditionals of deliberation can be deliberationally useful or deliberationally useless, that is, respectively, assertable or unassertable in a given context involving a particular deliberating agent. Following DeRose, we will illustrate this distinction by means of Allan Gibbard's famous poker game scenario.

Gibbard constructed his scenario as a challenge to propositional theories of conditionals that validate the Principle of Conditional Non-Contradiction (CNC), according to which two conditionals with the same antecedent and contradictory consequents, "If $\varphi, \psi$ " and "If $\varphi$, not $\psi$," cannot be simultaneously true (unless $\varphi$ is impossible). CNC is often perceived as an adequacy constraint on any theory that aspires to capture the meaning of a conditional in natural language. Incidentally, contexts of deliberation provide some extra motivation for endorsing CNC: apiece of advice of the form "If you take a cab, you will not be late for the concert" would have been of no use at all, if the sentence "If you take a cab, you will be late for the concert" had been an equally warranted assertion in the same context. Yet this is exactly what seems to be happening in Gibbard's story, which begins as follows:

Sly Pete and Mr. Stone are playing poker on a Mississippi riverboat. It is now up to Pete to call or fold. My henchman Zack sees Stone's hand, which is quite good, and signals its content to Pete. My henchman Jack sees both hands, and sees that Pete's hand is rather low, so that Stone's is the winning hand. At this point, the room is cleared. (Gibbard I98I: 23I)

For Gibbard's example to fly, we have to assume that Zack can be fully confident that Pete received his signals in good order. For instance, Pete might have used some secret signals, too, to confirm that he got Zack's message. 
Before Pete makes his decision, Zack sends Gibbard a message saying:

(9) If Pete calls, he will win.9

This is a warranted assertion, given that Zack knows that Pete is cheating, and hence will not call unless he has the winning hand. Yet (9) would constitute extremely bad advice for Pete if he were yet to decide whether to play or to quit. Similarly, even though owning a Maserati is very good evidence for being rich, and it is perfectly reasonable to believe that if Charlie buys a Maserati, he will be able to afford staying in a 5-star hotel, the sentence (8a) taken by Charlie as a piece of advice may lead to disastrous consequences. Since if Charlie, who does not own a Maserati and cannot afford a 5-star hotel, overheard (8a) and interpreted it as an instruction on how to get rich, it could lead him to take an enormous loan for a luxurious car. Such a decision would not only be irrational, but also rather destructive (though, as we have noted above, the sentence is too obvious to be ever interpreted in this way). Analogously, interpreting (9) as a guideline, Pete can end up calling despite his unfavourable position in the game. Unlike Charlie, however, Pete has a chance of not recognising (9) as a deliberationally useless conditional. If he overhears Zack's assertion, he may catch immediately what are Zack's reasons for asserting such a conditional. On the other hand, Pete might also begin to doubt that he interpreted Zack's signals correctly and come to think that he should call, because, given what Zack said, he is going to win.

By contrast, Jack's assertion:

(Io) If Pete calls, he will lose.

is backed up by his knowledge of exactly what cards the players have. It would not only be a piece of advice conflicting with the one from Zack, but also an extremely valuable one. In other words, Jack's ( $\mathrm{I}$ O) is a clear case of a deliberationally useful conditional. This observation alone is not helpful though, unless we can explain what makes indicatives like (9) pieces of bad advice. Given what Zack and Jack know about Pete, Mr. Stone's cards, poker game, and the whole situation, Pete's calling can be at the same time good evidence for his winning and good evidence for his losing, depending on the perspective of the speaker. ${ }^{\text {IO }}$ If Pete overhears (9) and (Io) (or, say, gets hold of the mobile phone

9 To serve as an example of deliberation, sentences asserted by Zack and Jack, originally in the past tense, had to be rephrased as future directed conditionals. This detail is of little importance for Gibbard's original argument.

Io One might be tempted to argue that, since Gibbard's scenario is asymmetrical in the sense that Jack's assertion seems to rely on much better grounds than Zack's, only the former is warranted. However, this is not enough to render Zack's conditional, (9), unassertable. Although Zack's reasoning is defeasible, because, for instance, Pete might have suddenly lost his interest in winning or he might have forgotten the information Zack communicated to him, Zack does not have reasons to believe that any of these unusual circumstances will occur and, although his knowledge is incomplete, he is not mistaken about anything that plays a role in the inference leading him to (9). This is why we have no basis for denying that (9) is assertable. Furthermore, Gibbard's story can be easily made symmetrical. For instance, one can consider a version of it in which the only thing Jack saw is that Mr. Stone's hand was very high, say, four of a kind. On this basis Jack could calculate that the probability of Pete's drawing a higher hand is less than 0.05 - highly unlikely, yet not entirely impossible. In this case, 
of a person for whom they were intended and reads the messages), assuming that he finds Zack and Jack equally trustworthy, he would be at a loss. Or, at least, he would be temporarily puzzled. Having received signals from Zack, he might be able to reconstruct the reasoning behind Zack's assertion, and having two seemingly conflicting conditionals to think about, he might well be able to conclude that his hand is weaker than Mr. Stone's. But if Pete's reasoning capacities, especially in a stressful situation and under the time pressure, are not up for the task, Pete might get very confused indeed. It is then not only the case that indicative conditionals can be misleading when uttered as conditionals of deliberation, but they can also conflict with each other. The presence of a strong evidential relation between a conditional's antecedent and its consequent is then not enough to guide a deliberating agent, that is, it is not enough to make a conditional assertable in a context of deliberation.

Implementing conditional dependencies through conditional probabilities into decision matrices was the first attempt to capture the impact of the agent's actions on the likelihood of their outcomes (Jeffrey I983). As such, it was one of the most important contributions of the evidential decision theory to our understanding of rational decision making, even if it turned out to be the source of its demise. As Joyce (I999: I46) points out:

Evidential decision theory requires agents to choose actions that provide them with good evidence for thinking that desirable outcomes will occur. While this is usually sound advice, there are decision problems in which the pursuit of good news can lead to less than optimal results. This happens when there is a statistical or evidential correlation between the agent's choices and the occurrence of certain desirable outcomes, but no causal connection between the two. When the evidential and the causal import of actions diverge in this way, the evidential theory tells decision makers to put the pursuit of good news ahead of the pursuit of good results. ${ }^{\text {II }}$

Gibbard's scenario illustrates the problem with evidential decision theory that Joyce seems to have had in mind. ${ }^{\mathrm{I2}}$ The indicative conditionals used in this scenario can provide a deliberating agent with conflicting advice, and one of them can lead a decision maker to the results which are highly undesirable. Does it mean though that we should never trust indicative conditionals in the contexts of decision making? Do deliberating agents have to base their choices on subjunctive conditionals to have a guarantee that they are guided by the expected results of their actions rather than by mere statistical correlations between these actions and some other desirable events? Like DeRose (2010), we are inclined to give a negative answer to these questions.

We mentioned above that deliberating agents are not likely to consider indicative conditionals such as those in (8) to be instructions. In the context of the Gibbard's scenario, too, as DeRose already pointed out, it would not be difficult to tell the good and the bad advice apart if both speakers' grounds for asserting the conditionals were known. Likewise, should the speakers of $(8 \mathrm{a}),(8 \mathrm{~b})$, or (9) realise that they are involved in the

Jack would still be justified in asserting (ro), but his assertion would carry a sense of uncertainty comparable to that of Zack's (9).

I I See also Lewis (I98I) for a similar critique of evidential or, as he calls it, non-causal decision theory.

I 2 This scenario could be construed as somewhat analogous to the famous Newcomb's problem (Nozick 1969), except it is more realistic, and hence more transparent. Arguably, a one-boxer would correspond to someone who interprets Zack's conditional as a piece of good advice (DeRose 20IO: 2I-22). 
contexts of Pete's, Charlie's, or Judy's deliberations, respectively, they would never assert their conditionals, or at least not without any further explanations. In most cases, neither decision makers, nor their advisers, as long as they have access to relevant information, have problems with interpreting a conditional like (8a), (8b), and (9) as deliberationally useless, while recognising (Io) as an example of a deliberationally useful conditional. Yet any non-causal decision theory rules that, as long as $\varphi$ is a good evidence for $\psi$, as in the cases of (8) or (9), agents should see to it that $\varphi$ is the case, whenever they want to achieve $\psi$. But this only means that decision theory missed something about the meaning of indicative conditionals which actual English speakers usually do not miss. This is why it is crucial to understand on what basis speakers decide that a conditional is assertable or unassertable in a particular context of deliberation, or, more generally, how people construct and interpret indicative conditionals. Only if we unravel the reasons why some indicative conditionals can behave so badly in contexts of deliberation, can we hope to construct a realistic decision theory which would not need to require people to rely on counterfactual conditionals.

Acknowledging that indicative conditionals can be systematically grouped into those that can be deliberationally useful and those that are deliberationally useless is obviously not an explanation of the phenomenon yet. It is, nonetheless, a valuable first step towards one. DeRose's own proposal is that future directed indicative conditionals "are deliberationally useless when they are based on backtracking grounds” (p. 28). Importantly, he did not argue that there is a class of backtracking conditionals and that conditionals from this class are unassertable in the context of deliberation. What he claimed is that in some cases, and in the cases of deliberationally useless conditionals in particular, backtracking reasoning is essential in providing grounds for the assertion. Indeed, one could construe Zack's perspective in the poker game scenario as involving some form of reasoning "back in time": Zack supposes that, at a point in time $t_{i}$, Pete calls, and then he reasons from that hypothetical event at $t_{I}$ back to $t_{i-I}$ to find out what preceded Pete's calling. Zack realises that, at $t_{\mathrm{i}-\mathrm{I}}$, Pete knows what cards his opponent, Mr. Stone, has, so he would only call in the situation in which his cards are stronger than Stone's. In other words, Pete would only call if he were to win. By contrast, Jack's reasoning does not require any steps up the time stream: a simple transition from the assumption of Pete's calling at $t_{i}$ to his losing at $t_{i+1}$ is all there is to it.

DeRose has identified an important difference between the kind of reasoning that underpins Zack's assertion of (9) and Jack's grounds for (Io). However, although DeRose's analysis of Zack's perspective is correct, he does not fully explain why (8a), (8b), or (9) happen to be deliberationally useless. In fact, as we shall argue, the presence of backtracking reasoning is not sufficient to render a conditional unassertable in a context of deliberation and neither is it necessary. In what follows, we will show that a whole class of deliberationally useful conditionals notably depends on reasoning back in time and that there are deliberationally useless sentences which do not.

\section{BEYOND BACKTRACKING}

In Section 2, we conceded that the difference between the two conditionals in the Gibbard's scenario can be analysed in terms of different causal structures providing grounds for their assertions. We are of the opinion, however, that it is not the 
backtracking itself that makes an indicative useless in a context of deliberation. In fact, there is a whole class of indicatives that happen to be deliberationally useful even though they essentially depend on backtracking grounds. These are deontic indicative conditionals that we have introduced at the beginning of the paper, i.e., sentences falling under the schema "If an agent $A$ wants $\psi, A$ should $\varphi$ ":

(7) a. If you want the sauce to taste better, you can add some fresh herbs.

b. If they don't want to be late for the concert, they should take a cab.

c. If I don't want to move to London, I shouldn't accept this job.

d. If you don't want this plant to die, you should water it.

They express precisely the kind of dependencies between actions and states of affairs that are relevant for decision making, and hence, as we have argued, they fall under the definition of conditionals of deliberation. Moreover, as long as they are well justified, these sentences tend to constitute rather good pieces of advice.

Though they are not backtracking conditionals per se, and the reasoning pattern involved is slightly different than in the cases we discussed earlier, their dependence on reasoning back in time is crucial, which is exactly what DeRose identified as the source of deliberational uselessness (DeRose 20I0: 28). The antecedents of the above conditionals are propositional attitude reports, and as such they do not seem to be preceded by whatever is expressed by their consequents. But to figure out what should be done in order to fulfil the wishes or plans reported by the antecedents, one has to start with the objects of those propositional attitudes. For instance, the speaker of the following sentence:

(I I) If Malcolm wants to have a well-paid job, he should study law.

considers a possibility that Malcolm finds a well paid job at the time $t_{n}$ and reasons back in time to $t_{n-k}$ to find out what could make this happen, that is, which study programme would most likely lead to a career providing good pay. Obviously, there is more than one possibility, but we can take into account Malcolm's own preferences and inclinations to narrow down possible options. For instance, he might be really fond of history and politics, while not being particularly tech-savvy, so an engineering programme would not suit him, nor interested in physical sciences at all, which makes a medical school a dubious choice, too. Consequently, we can arrive at the conclusion that, since lawyers tend to earn good money and law seems to be a career path at which Malcolm has a chance to succeed, he should study law. More precisely, the following procedure is a plausible reconstruction of the reasoning that may lead a speaker to the assertion of (II):

I. Assume the antecedent of a conditional, $\gamma$ (e.g. "Malcolm wants to have a well-paid job");

2. "Extract" the embedded sentence $\psi$ ("Malcolm has a well-paid job") from the attitude report $\gamma$ : "an agent $A$ wants $\psi$ ty to be the case" ("Malcolm wants "Malcolm has a wellpaid job' to be the case");

3. Assume that $\psi$ holds at a future time $t_{n}$;

4. Reason back in time, from $t_{n}$ to $t_{n-k}$, to see what at $t_{n-k}$ needs to be the case to make $\psi$ be the case. Given the relevant background knowledge, choose which of the available 
options is the best way for the agent $A$ to achieve $\psi$, and call it $\varphi$ ("Malcolm studies law").

5. Embed $\varphi$ in a deontic statement $\xi$ attributing $A$ an obligation to see to it that $\varphi$ is the case, as in: "an agent $A$ should $\varphi$ " ("Malcolm should study law").

6. Assert "If $\gamma$, then $\xi$ " (II).

Although backtracking involved in the production of a deontic conditional of deliberation is only a step in the whole reasoning process underpinning the assertion, there is no doubt that, if the speaker follows the above procedure, their assertion significantly depends on backtracking grounds. Treating the presence of backtracking as a symptom of deliberational uselessness would then exclude deontic conditionals, a vast class of sentences that can often help a deliberating agent, from the class of conditionals which are deliberationally useful. ${ }^{\mathrm{3}}$ More importantly, the above example shows that there is nothing particular about reasoning back in time that renders conditionals such as (8) or (9) deliberationally useless. Saying that these sentences depend on backtracking reasoning, even if true, does not explain why they are useless. Moreover, such a diagnosis seems to rest on an assumption that all conditionals of deliberation can be analysed in causal terms.

I have noted at the beginning of this paper, quoting authors like Joyce, Lewis, or Gibbard and Harper, that deliberating agents, and, consequently, decision theorists modelling these agents' reasoning processes, are primarily interested in causal relations between actions and their outcomes. I do not subscribe to a view, however, that only causal conditionals can be deliberationally useful. There are conditionals of deliberation whose usefulness does not rest on any causal considerations, and this is an example of such a conditional:

(I2) If you get a Golden Retriever, it will be a friendly dog.

Someone who is looking for a breed of dog that would guarantee the animal to be a good family pet, may appreciate such a tip. What the above sentence is meant to convey is the presence of a relationship between the particular breed and the desirable qualities a dog of this kind can be expected to have. (I2) is clearly a case of a conditional of deliberation, not just one of those conditionals that can be asserted in the context of deliberation: its consequent expresses a proposition whose truth value is of interest to the agent, and the antecedent expresses a proposition whose truth value the agent can directly influence. In other words, the conditional's consequent provides reasons to take the action that the antecedent is about. Moreover, the same piece of advice can be phrased as an explicit conditional of deliberation: "If you want a friendly dog, you should get a Golden Retriever." However, what is relevant for a decision making agent here is not the information

I3 A referee has pointed out that a speaker might arrive at (II) by identifying Malcolm's desire and using general knowledge about means-end relations, without relying on backtracking at all. Although this is a valid observation, it does not threaten the claim that the dependence on backtracking is not sufficient for a conditional to be deliberationally useless as long as the above procedure accurately represents, as I believe it does, how some speakers come to assert a deliberationally useful deontic conditional in some contexts. Even if such situations turned out to be rare, our claim that backtracking is not diagnostic of deliberational uselessness is still justified. 
about any purported causal link between buying the Golden Retriever and the dog's friendliness; buying the dog is not what makes it friendly. What the conditional conveys is that the Golden Retriever belongs to a set of dogs that have the qualities sought out by the decision maker. To put it differently, the assumption that the dog is the Golden Retriever allows us to infer, or, to be more precise, predict that it will be a friendly family pet, which is the outcome that our decision maker desires, but this inference is not based on any causal considerations, but rather on a set membership (or set-subset) relation.

Since, as it turned out, deliberationally useful conditionals do not always need to be based on causal inferences, it should not be surprising that backtracking is not necessary for an indicative conditional (whose surface structure allows it to serve as a conditional of deliberation) to be rendered deliberationally useless. To make this point clear, imagine two fashion enthusiasts, Felicity and Felix, searching for something extraordinary at a thrift shop. Felix found a weird looking suede purple jacket, but Felicity is rather sceptical that Felix would be confident enough to wear such extravagant attire. Two days later, however, much to her surprise, Felicity saw Felix sporting that strange, purple suede piece of clothing. As she realised that he is more self-assured than she has suspected, Felicity asserts:

(13) If Felix wears that jacket, he will be able to wear just about anything.

Wearing a particular jacket is not really an action that would help an aspiring fashion aficionado to build up self-confidence necessary to wear whatever extravagant costume he fancies. It would be, however, good evidence for Felix's being sufficiently self-assured for that purpose already. The above sentence is a deliberationally useless conditional, similar to those in (8). Imagine that, by a strange coincidence, Felicity did not see Felix, but someone else who, from a distance, looks very much like her friend and who happened to wear a purple suede jacket strikingly similar to one that Felix found in the thrift shop. Now, Felicity might assert ( $\mathrm{I}_{3}$ ) without realising that Felix can hear her, that is, without realising that she might be asserting the conditional in a context in which Felix is deliberating if he should be wearing the jacket. If he is only a beginning fashionista who does not feel comfortable in some of the more extravagant clothing, but who is working on building his self-assurance by making small steps in the direction he desires, namely, being able to wear anything he imagines, no matter how unusual, suddenly making a jump might not be the right thing to do. He should not take ( $\left.\mathrm{I}_{3}\right)$ as a piece of advice, and Felicity should not assert this conditional in a context in which it can be taken as such. This conditional is deliberationally useless, yet no backtracking grounds are present. A person's disposition to feel comfortable in extravagant clothing is not a cause of their ability to wear a particular jacket. ( $\mathrm{I}_{3}$ ) is another example of a statement expressing an inference based on a set membership relation rather than a causal link.

What is then a difference between deliberationally useful conditionals like (I), (IO), or (I2), on the one hand, and deliberationally useless conditionals like (8), (9), or (I 3), on the other? I have shown that the presence of backtracking reasoning is neither sufficient nor necessary to make a conditional unassertable in the contexts of decision making. In the following sections I will provide an alternative account of this phenomenon. Before I can unfold my own explanation of what renders a conditional deliberationally useless, I need to ponder over a more fundamental question, namely, what it is exactly that people assert when they decide to phrase their thoughts as indicative conditionals. 


\section{WHAT DO WE ASSERT WHEN WE ASSERT A CONDITIONAL?}

A decision theory, be it Jeffrey's evidential framework or the causal framework of Joyce, relies on probabilities of a particular kind, i.e. conditional probabilities or probabilities of subjunctive conditionals, respectively, to calculate the expected utilities of various actions. One could argue that a linguistic form of a sentence we use to express thoughts about the relevant conditional dependencies is of little importance: as long as we use probabilities of the right kind whenever we need to calculate expected utilities of various options described by the antecedents of conditionals, be it indicatives or subjunctives, we should be safe, right? Well, not exactly. The way we describe the relationships between actions and other states of affairs can make a difference; conscious decision making happens, after all, in a language. People do not draw decision tables - philosophers who want to model rational decision making do. Instead, deliberating agents think in terms of conditional sentences, or they listen to tips or warnings that are conditional in form, and these conditionals provide us, the modellers, with information necessary to construct and analyse the decision problems in a more abstract way. We need to interpret these conditionals before we can draw our decision matrices and assign probability values to outcomes that depend on various actions.

So what is it that we assert when we assert that if $\varphi$ then $\psi$ ? What kind of information do we convey when we choose to phrase a thought as an indicative conditional? And what is it that a hearer learns from such an assertion? Up until now we have only been concerned with assertability or unassertability of certain conditionals in contexts of deliberation, but we have avoided the controversial issues of their truth conditions and their acceptability conditions. To push ahead with the discussion, however, we need to touch upon the issue of their meaning. After all, the minimal requirement for the assertability of a sentence $\xi$ for a speaker $S$ is that $S$ believes (i.e. accepts) that $\xi$ (Grice 1989; Bach 2008). ${ }^{\mathrm{I}}$ Since it does not seem to be possible to disentangle the question of a sentence's acceptability conditions from the question of its interpretation, let us ponder over the acceptability of indicative conditionals first.

Intuitively, when we need to decide whether to accept "If $\varphi$ then $\psi$ " we suppose $\varphi$, that is, we imagine that $\varphi$ is the case, and ask ourselves if $\psi$ holds under that supposition, or if $\psi$ becomes true when $\varphi$ is. Frank Ramsey captured this intuition in what came to be known as the Ramsey Test:

If two people are arguing 'If $p$ will q?' and are both in doubt as to $p$, they are adding $p$ hypothetically to their stock of knowledge and arguing on that basis about $q$ : so that in a sense 'If $p, q$ ' and 'If $p\urcorner$,$q ' are contradictories. We can say they are fixing their degrees of belief in q$ given $p$. (Ramsey I929/I990: I55)

Since in its original phrasing, the Ramsey Test leaves a lot of space for interpretation, it inspired the development of at least two different families of accounts of indicatives. So different, in fact, that their proponents disagree about issues as fundamental as indicative conditionals' truth-aptness. Robert Stalnaker (I968, I975), for instance, developed Ramsey's idea into a full-fledged truth-conditional semantics for indicative conditionals.

I4 In fact, the majority of normative accounts of assertion impose much stronger requirements than a mere belief, such as knowledge (see, e.g., Williamson 2000) or rational credibility (Douven 2006). 
On this account, a conditional, "If $\varphi$, then $\psi$," is true if and only if $\psi$ is true in the closest possible $\varphi$-world, i.e. the closest possible world in which $\varphi$ holds, where "a possible world is the ontological analogue of a stock of hypothetical beliefs" Stalnaker (I968: IO2). A speaker might be, of course, mistaken about which world is the actual world, and hence about the truth value of any given conditional. Nevertheless, what a speaker communicates when they assert a conditionals can be reconstructed in terms of possible worlds, too. Since propositions can be represented as sets of possible worlds, a speaker's beliefs, that is, the propositions accepted by that speaker can be thought of as determining which world (or worlds) they consider to be candidates for the actual world. The intuition behind the closest possible antecedent-world corresponds to Ramsey's idea of the minimally revised stock of beliefs. When a speaker asserts a conditional, on this account, they communicate that the consequent, $\psi$, holds in a world ${ }^{\mathrm{I}} 5$ they imagine to be exactly like our actual world except that the conditional's antecedent, $\varphi$, holds in it.

By contrast, other authors take the Ramsey Test to suggest a method of fixing one's degrees of belief in a conditional, that is, one's subjective probability that a conditional holds. What evaluating an indicative conditional amounts to then is calculating the conditional probability of that conditional's consequent given its antecedent. This idea can be formally expressed as what became known simply as the Equation: $\operatorname{Pr}$ ("If $\varphi$, then $\left.\psi^{\prime \prime}\right)=\operatorname{Pr}(\psi \mid \varphi)$. Although it is intuitively appealing and, to a great extent, supported by empirical data on how people reason with indicative conditionals, ${ }^{16}$ endorsing the Equation turns out to come at a cost.

As David Lewis's 1976 famous triviality results suggest, one cannot maintain the Equation and a truth-conditional account of conditionals at the same time. In response to Lewis's proof, many authors rejected the idea that indicative conditionals have truth conditions at all and endorsed a non-propositional view on their meaning (e.g. Edgington I995; Bennett 2003). ${ }^{17}$ On these accounts, the meaning of an indicative conditional, no longer understood as its truth conditions, but instead as its acceptability conditions, is exhausted by the conditional probability of that conditional's consequents given its antecedents. This means that whenever a speaker asserts "If $\varphi$, then $\psi$," all they literally say is that $\operatorname{Pr}(\varphi \mid \psi)$ is high.

The Ramsey Test thus understood seems to capture the intuition that whenever we wonder if it is the case that, for instance, Jim will be upset if he gets fired, we imagine what would happen in a situation in which Jim gets fired. If the rest of our beliefs suggest that Jim is more likely to feel relieved since he has been thinking about quitting for a long time yet had no guts to face his boss and file a resignation, we will not accept the conditional:

(I4) If Jim gets fired, he will be upset.

I 5 Or in a set of worlds, cf. Lewis (1973).

I6 In the psychology of reasoning, there is a lot of evidence showing that people judge the probability of a conditional to be the corresponding conditional probability (see, e.g., Evans and Over 2004), though recent results by Skovgaard-Olsen et al. (20I6) suggest that this is only the case when $\varphi$ is positively relevant for $\psi$

I7 Lewis's proofs rely on a number of assumptions, other than the Equation and conditionals' truthaptness, that may also be contested (see, e.g., Douven 2016: 7I-77). Nonetheless, questioning the very possibility of indicative conditionals expressing propositions turned out to be the most common response. 
In fact, performing the Ramsey Test will lead us to accepting its negation, assuming, as it has often been argued (e.g. Edgington I995: 283), that negating a conditional amounts to negating its consequent: ${ }^{\mathrm{I}} 8$

(I 5) If Jim gets fired, he won't be upset.

Yet the Ramsey Test, be it under the possible worlds or probabilistic interpretation, does not always do justice to our intuitions about the acceptability of indicative conditionals.

The problems arise when we already believe that the antecedent and the consequent of a conditional are both true. Consider the following sentence:

(I6) If Daniel Kahneman was awarded the Nobel Prize in Economics, London is the capital of the United Kingdom.

This sentence strikes us as odd, because Daniel Kahneman's achievements, no matter how great they are, cannot have anything to do with London's status as the country's capital. Yet, given that we already believe that Daniel Kahneman received the Nobel Prize in Economics and that London is the capital of the United Kingdom, the conditional passes the Ramsey Test (on its most popular interpretations). Consequently, the probabilistic account renders the conditional highly acceptable, because the conditional probability of London being the capital of the UK given that Kahneman was awarded the Nobel Prize in Economics is very high. In fact, if we assign probability I to the consequent of a conditional, and the probability of that conditional's antecedent is greater than $\mathrm{o}$, the conditional probability also has to equal I, regardless of whether given conditional would be a reasonable thing to say. If we want to analyse this sentence in terms of Stalnaker's possible worlds semantics, we encounter the same problem: the nearest possible world in which Kanhemann was awarded the Nobel Prize in Economics is our actual world. Since it is also true in the actual world that London is the capital of the UK, (I6) is true.

This might not seem very problematic yet. We could repeat the many philosophers who adopted the Gricean solution to the problem of odd truths (Grice I989), that there are many true sentences that will simply never be assertable, and this is not something a semanticist should worry about. But is believing conditionals that we would never assert really so harmless? Let us return to the example of Jim who would certainly not get upset if he lost the job he hated. Imagine now Jim at a later point in time, when he works for a completely different company. Much to his relief, he indeed got fired from his previous job, but he quickly found a new and exciting appointment, and he is now fully satisfied with his position. Nevertheless, Jim is extremely upset, depressed in fact, but this has nothing to do with his professional life. The reason why Jim is going through an emotional breakdown is a divorce he has a difficulty coping with. In these circumstances, the present tense version of (I4):

(I7) If Jim got fired, he is upset. 
does not seem to be acceptable at all, yet our strong belief in the antecedent and in the consequent of the above conditional would make it pass the probabilistically interpreted Ramsey Test with flying colours. The possible-worlds interpretation of a conditional yields similar results: since both "Jim got fired" and "Jim is upset" are true in the actual world, the conditional (I7) is true. Unlike (I6) though, this conditional does not seem to be a bizarre thing to say; prima facie, there is nothing obviously wrong with it. Yet, if asserted, it is bound to lead a hearer to a false belief that it was the dismissal from the job that is the reason for Jim's distress. The conditional seems to convey some sort of a causal relationship between the two facts. Furthermore, this problem goes beyond the assertability: accepting a conditional just because both of its clauses are true and the corresponding conditional probability is high will lead to the same kind of an unwarranted belief in a connection between the conditional's antecedent and its consequent. ${ }^{\text {I9 }}$

To see that the problem does not boil down to the unassertability of sentences that sound odd, picture a conversational context in which someone asserts a conditional suggesting a connection between its antecedent and consequent whose existence we would not accept, for instance:

(I 8) If Tom does not practice headstands everyday, he will never learn to levitate.

We may safely agree that Tom will not learn to levitate whatever he does, simply because levitation is physically impossible. The consequent of the above conditional is then true. Let us also assume that we know Tom sufficiently well to be highly confident that he is not into any sports or exercises whatsoever. He is not even able do a headstand, let alone practise it on an everyday basis. This makes the antecedent of the above conditional true as well. Like (I6) and (I7) discussed above, (I 8) passes the Ramsey Test on both possible worlds and probabilistic account. One could argue then that (I8) is not assertable for us on Gricean grounds, since we are entitled to assert something stronger, namely:

(I9) Tom will not practice headstands everyday, and he will never learn to levitate.

Yet we can imagine some spiritual leader, let us call him Misty, who would try to convince us, the sceptics, that levitation is within reach for those of us who foster their inner powers by regularly standing on their heads. Suppose that Misty asserts (I 8). Are we in a position to disagree with him? If all this sentence means is that it is highly probable that Tom will never learn to levitate given that he does not practice headstands, the answer is: no. We cannot disagree with Misty and, moreover, we should find (I 8) perfectly acceptable. Still, the following dialogue seems to be a quite likely outcome of our meeting with Misty:

(20) Misty: If Tom doesn't practice headstands everyday, he will never learn to levitate. Scept: Nonsense, he won't learn to levitate even if he does. Handstands will not make him able to fly!

I9 Cf. the Principle of Epistemic Hygienics proposed by Douven (2010), who argued that accepting unassertable sentences may mislead our "future selves." 
We find (I 8) disagreeable, because someone who asserts it seems to communicate their belief in some kind of a causal relationship between the content of the antecedent and the content of the consequent. One could express this relationship, for instance, in the following way:

(2I) Tom will never learn to levitate, because he does not practice headstands everyday.

Someone who sincerely asserts (I 8 ) is likely to believe (2I), too. Setting aside whether (2I) is a paraphrase of the conditional, its logical consequence or whether it is pragmatically implied or implicated, ${ }^{20}$ it is by no means a belief that an agent endorsing (I9) should be committed to. One could perhaps try to turn this point around and argue that (I8) is actually an infelicitous way to express (2I). Since we reject the latter, we should not accept the conditional either. But this would make at least some conditionals, namely those that express causal relations, constitute a special class of conditional sentences whose meaning is indeed neither exhausted by the corresponding conditional probability, nor determined by the truth value of their consequent in the nearest antecedent-world.

As we have shown above, not all indicative conditionals convey the presence of a causal link, but many, if not all, seem to indicate some kind of a connection between their antecedents and consequents. It is a plausible hypothesis that interpreting a conditional amounts to reconstructing what that connection really is. Imagine that someone asserts:

(22) If some form of life is found on Mars, Bill can retire.

If you do not know who Bill might be, at least at the first glance, the sentence might appear somewhat nonsensical. But if you only think about it for a little while, you will probably be able to come up with contexts in which (22) would be a perfectly reasonable thing to say. Bill might be, for instance, an astrobiologist approaching his retirement, who devoted his life to the search of extraterrestrial life forms. Given the recent evidence indicating that there is water on Mars, he might have decided to postpone his retirement until Curiosity's mission is over.

The way we deal with a conditional when we are not provided with the context of its utterance resembles the interpretation of sentences with indexicals. If, as in the case of (22), we have no information about the context in which, for instance:

\section{(23) I have lost it.}

has been uttered, and in particular we know neither who said it, nor what "it" refers to, we do not learn much from this sentence. It is not to say, however, that we learn nothing at all. We can be sure that whoever asserted it used to be in possession of something, but he or she does not have it any more. Moreover, the choice of the verb, "lost," is also telling. It conveys that what "it" refers to was not sold or given away by the speaker who most likely did not intend to be deprived of it. Even if what we do when trying to interpret sentences

20 Note that it is not entirely clear how the fact that conditionals suggest the existence of a connection between their antecedents and consequence could be explained by pragmatics alone (cf. Douven 20I7). 
like (23) or (22) is mere speculation, this speculation follows certain rules. One could argue that these rules are precisely what determines the meaning of a linguistic expression.

The interpretation of indicative conditionals advocated in this paper treats conditionals as highly context sensitive. What depends on the context, and on the epistemic states of a speaker in particular, may be construed as the way one proceeds with the Ramsey Test. I noted earlier that a speaker is warranted in asserting a conditional if the conditional passes the Ramsey Test in the first place. But, as the examples such as (I8) and (I7) or the examples of deliberationally useless conditionals like (9) show, we cannot rely on the Ramsey Test alone. It seems clear that the antecedent and the consequent should be somehow connected, that the antecedent should play a role in arriving at the consequent. We shall argue that the very procedure that brings us from the assumed antecedent to the consequent is the key to understanding what conditionals are and why some of them can harm a deliberating agent.

\section{INFERENTIAL CONDITIONALS AND THE SOURCE OF THE USELESSNESS}

In Section (4), I argued that when interpreting a conditional, even if no information about the context of its utterance is provided, we tend to see (or, if it is not salient, we try to find) a connection between that conditional's antecedent and its consequent. In this section, I will show that finding out what that connection might be is crucial for our understanding of why some indicatives are unassertable in the context of deliberation while others can serve as perfectly reasonable pieces of advice.

The examples of deliberationally useful and deliberationally useless conditionals discussed in this paper, and the pair of (9) and (Iо) from the Gibbard's scenario in particular, provide some evidence for the claim that indicative conditionals do not constitute a homogeneous class: even if two conditional sentences share the surface structure, they may have very different assertability conditions, if not more. This should not come as a surprise. Even though the vast majority of philosophical theories of conditionals are attempts to provide a unified account of all sentences of the form "If $\varphi$, then $\psi$," at best making a distinction between indicatives and counterfactuals or subjunctives, the situation in linguistics seems to be different altogether. For instance, it is customary in linguistics to distinguish between content conditionals, inferential conditionals, and speech-act conditionals, depending on the domain of the conditional relation between their antecedents and consequents (see, e.g., Sweetser I990; Declerck and Reed 200I; Dancygier and Sweetser 2005). In speech-act conditionals, the antecedent provides a condition for the performance of the speech act represented in the consequent, for instance:

(24) a. If you are hungry, there are biscuits on the sideboard.

b. If you don't mind me asking, how old are you?

c. If I don't see you before Thursday, have a good Thanksgiving!

There is a great variety of speech acts, and thus of speech act conditionals, but what they all seem to have in common is the fact they can be paraphrased by "If [the antecedent holds], then let us consider that I perform this speech act (i.e. the one represented by the consequent)" (Sweetser I990: I2I). These sentences will not concern us 
here, however, since only the remaining two classes of conditionals are relevant for our purposes.

Content conditionals are said to indicate that the realisation of the state of affairs or event described by the antecedent is a sufficient condition for the occurrence of the state of affairs or event described by the consequent, e.g.:

(25) If Mary goes, John will go. (Sweetser I990: I I4)

The connection between the antecedent and consequent of a content conditional can often be thought of as causal: Mary's going may be what will cause John to go as well. Inferential conditionals, by contrast, are said to express relations between epistemic states. The antecedent of an inferential conditional can be construed as a premise of an argument: knowledge of the truth of the antecedent is sufficient condition for concluding the truth of the consequent, for instance:

(26) If she's divorced, (then) she's been married. (Sweetser I990: II6)

The above sentence is an example of a tautological conditional, in which the consequent follows from the antecedent in virtue of the meaning of the terms they involve. Not all inferences that can be expressed by inferential conditionals are indefeasible. Consider the following example:

(27) If my bicycle saddle is wet, it must have been raining.

Here, knowing that the saddle is wet allows the speaker to infer that it has been raining, because rain appears to be the most likely (even if not the only conceivable) cause of wetness of the saddle.

It could be argued that many - if not all - indicative conditionals can be analysed in terms of inferential relations between their antecedents and consequents. In fact, many sentences that linguists classify as content conditionals can be construed as expressing inferences as well, just, in the case of content conditionals, these inferences are of a different kind, namely, causal. ${ }^{21}$

The idea that a conditional expresses an inferential relation is not unique to linguistics. Among the advocates of the inferential view one can find John Stuart Mill:

'If $A$ is $B, C$ is $D$,' is found to be an abbreviation of the following:

'The proposition $C$ is $D$, is a legitimate inference from the proposition $A$ is $B$.' . . a conditional proposition is a proposition concerning a proposition; the subject of the assertion is itself an assertion. (Mill I 843: II)

2I Our understanding of the notion of an inferential conditional diverges here from that used in linguistic literature, because we take it to denote a broader concept (cf. Verbrugge 2007; Verbrugge et al. 2007). This is why, from our philosophical perspective, it is difficult not to perceive many of the so-called content conditionals as expressing an inferential connection between their antecedents and consequents, too. These terminological discrepancies have no bearing on the main point of the paper. 
Arguably, this line of thinking about conditionals can be traced back to a stoic logician, Chrysippus (Sanford I989), and seems to have been endorsed by later philosophers such as Mackie (I973) or Ramsey (I929/I990: I 56):

In general we can say with Mill that 'If $\varphi$, then $\psi$ ' means that $\psi$ is inferrible from $\varphi$, that is, of course, from $\varphi$ together with certain facts and laws not stated but in some way indicated by the context.

The idea that the clauses of a conditional should be inferentially connected has been recently revived. For instance, Spohn (2OI3) and Skovgaard-Olsen (2OI4) construed indicatives as expressing reason relations. Independently, Krzyżanowska et al. (2013, 20I4), Douven (20I6), and Douven et al. (20I8) followed the above cited linguists in analysing conditionals as corresponding to arguments or, to put it differently, expressing inferences from their antecedents to consequents. Unlike our predecessors, in Krzyżanowska et al. (2013, 2014), we base our analysis on an observation due to Douven and Verbrugge (20IO) that the inferential relation connecting the premises and the conclusion of an argument expressed by a conditional does not always have to be deductive. Douven and Verbrugge argued that inferential conditionals can be classified analogously to how inferences are, ever since Peirce's seminal work on arguments (see, e.g., Burch 20I4), typically classified. The first distinction that this typology makes is between certain and uncertain inferences. Certain inferences coincide here with deductively valid ones. Uncertain inferences, by contrast, are defeasible, that is, they can only guarantee that the conclusion is very likely, given the truth of the premises. The uncertain inferences can be further divided into inductive inferences, loosely defined as inferences whose conclusions are based on certain statistical information, and abductive inferences, understood here as inferences to the best explanation. This typology led Douven and Verbrugge (2010: 303-304) to the following definition of an inferential conditional:

Definition 4. A sentence "If $\mathrm{P}$, then Q" is a (contextual) deductive inferential (DI, for short), inductive inferential (II), or abductive inferential (AI) conditional if and only if $\mathrm{Q}$ is, respectively, a deductive, inductive, or abductive consequence of $\mathrm{P}$ (or of $\left\{\mathrm{P}, \mathrm{P}_{\mathrm{I}}, \ldots, \mathrm{P}_{\mathrm{n}}\right\}$, with $\mathrm{P}_{\mathrm{I}}, \ldots, \mathrm{P}_{\mathrm{n}}$ being background premises salient in the context in which "If $\mathrm{P}$, then $\mathrm{Q}$ " is asserted or being evaluated.)

Taking the above classification of conditionals for granted, in Krzyżanowska et al. (2014) we proposed a new analysis of the Gibbard's Poker game scenario, defending the view that conditionals are truth-evaluable, though their semantic values are highly context-sensitive. What needs to be picked up from the context, on this account, are not only the background premises, but also the kind of an inference that connects the conditional's antecedent and its consequent. On this account, "If $\varphi, \psi$ " is true if and only if a speaker $S$ can infer $\psi$ from $\varphi$ in conjunction with $S$ 's background knowledge, which we will denote here as $\mathrm{B}$, where this inference can be deductive, abductive, inductive, or it can involve a mixture of steps of different kinds. Unless $\psi$ is a consequence of $\varphi$ on its own, as it is in the case of a tautological conditional (26), two additional conditions must be satisfied: (i) $\psi$ cannot follow from B alone, and, (ii) $\varphi$ must be deductively consistent with B. To account for the empirical evidence suggesting that, in some cases, people 
find the truth values of indicative conditionals undetermined (see, e.g., Evans and Over 2004), in a more recent paper, Douven (2017) proposed a revision of the above semantics. On the new version of the account, a conditional is false when the argument connecting $\varphi$ and $\psi$ is weak, or when there is a strong argument from $\varphi$ to the negation of $\psi$, and as neither true nor false if there is no connection between its antecedent and consequent at all.

In this article, I am not addressing the question of truth-evaluability of indicative conditionals, and hence I am not going to debate nor defend the tenability of the truthconditional "inferential" semantics. The lesson we want to draw from it does not depend on any particular resolution of this highly controversial issue. Instead, we are going to focus on those aspects of the "inferential" view on conditionals which can contribute to a better understanding of the phenomenon of deliberationally useless conditionals. On the "inferential" account, what "If $\varphi, \psi$ " conveys does not depend merely on the truth values of $\varphi$ and $\psi$, nor is it exhausted by the subjective conditional probability, $\operatorname{Pr}(\psi \mid \varphi)$. What a speaker communicates when they assert an indicative conditional, that is, the information that such a sentence conveys, depends on what one could call the speaker's epistemic intentions, which, in turn, determine the reasoning processes that lead the speaker from the assumption of $\varphi$ to the conclusion of $\psi$. To see that this approach will allow us to shed some new light on the problem of deliberationally useless conditionals, let us have a closer look at its application to Gibbard's argument.

In Krzyżanowska et al. (20I4), we analyse Zack's assertion of (9) and Jack's (10) as expressing inferential connections of different types. Jack's assumption of the antecedent, "Pete calls," together with the background information on the distribution of cards and on the rules of the game leads him to the conclusion expressed by the consequent: "Pete will lose." It does so, because, given Jack's knowledge, Pete's losing follows deductively from the supposition that he called. Zack's assertion, on the other hand, is based on, as DeRose quite correctly observed, backtracking reasoning, that is, reasoning on what precedes the state of affairs expressed by the antecedent. But it is not the fact that Zack's reasoning tracks events back in time that makes a difference here. Under a supposition that Pete calls, Zack considers the reasons for Pete's decision to call rather than its consequences. He realises that, given Pete's character, his motivation to win, understanding of the rules of the game, and, most importantly, the fact that he is cheating, the best explanation of Pete's calling would be that he has the winning hand. To assert the conditional, Zack only needs to make one more inferential step. He infers "Pete will win" deductively from the supposition that he calls and from what is, given Zack's background knowledge, the abductive consequence of that supposition, namely, Pete's having the winning hand.

The other examples of deliberationally useless conditionals that we discussed earlier can be analysed in an analogous way. Consider the example (8a). We have seen that Charlie's buying a luxurious car would be good evidence for his being rich enough to be able to afford a 5-star hotel. Yet, despite the presence of a rather strong argument from the conditional's antecedent to its consequent, the conditional is not a good recommendation for Charlie. Finding out that the antecedent is true would allow us to infer that Charlie is rich, but making the antecedent a reality will not bring about Charlie's wealth. The reason for this is that the inference from "Charlie buys a Maserati" to "Charlie will be able to afford staying in a 5-star hotel" involves an abductive step: Charlie's being wealthy 
is the best explanation of his buying a Maserati, and hence the conditional is useless qua piece of advice. By contrast, a conditional expressing an inductive inference could be very helpful, for instance:

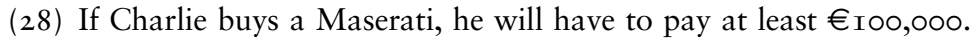

This conditional is deliberationally useful, because it provides a deliberating agent with information about the consequences of buying a Maserati, should he ever consider that. (28) may be asserted, for instance, by someone who is looking at a Maserati catalogue and observes that the prices begin at around $€_{\mathrm{I} O 0,000}$. The consequent of this conditional follows then deductively from the antecedent together with the speaker's background knowledge about the prices of such cars (and an additional assumption that only new cars need to be taken into consideration). (28) might be a very useful piece of advice for Charlie if he is, say, someone who just expressed his wish to own a Maserati, but who has done no research yet and does not know if he could afford such a car at all.

The example (8b) is no different: Judy's being extremely stressed is a symptom of an upcoming talk, not the means to an end. Again, the conditional is deliberationally useless since its consequent explains the antecedent instead of stating what the antecedent can bring about. A speaker who knows Judy well and heard her talks before might infer, on the basis of her previous experience and in combination with the inference expressed by (8b), that Judy will speak too quickly for her talk to be intelligible and, consequently, assert:

(29) If Judy is so stressed, she will speak too quickly to be intelligible.

Such a conditional can be deliberationally useful. Given Judy's academic aspirations, speaking too quickly for her talk to be intelligible is an undesirable outcome, hence it might motivate her to work on some methods of relaxation (whether the agent has a power over the truth value of the antecedent in this particular case is a different issue altogether). ${ }^{22}$

The "inferential" account of conditionals clearly is at least as good as DeRose's in recognising those indicative conditionals that become deliberationally useless in the contexts of deliberation, even though they are perfectly assertable otherwise. However, all

22 It is important to note that, although utilities play an important role in decision making, they do not necessarily affect the assertability of any given conditional of deliberation. The speaker does not need to be familiar with the agent's utilities to be able to provide arguments for or against particular actions. In fact, they might assert a whole series of conditionals that point in different directions. For instance, someone deliberating over a purchase of a possibly expensive car might be interested in the following inductive inferential conditionals: "If you buy a Maserati, you will spend a lot of money on a car which will probably break down all the time. On the other hand, if you buy a Maserati, you will have a luxurious vehicle that all your neighbours will be jealous about. But if you buy a Toyota, you will save a lot of money and have a more reliable car ..." All of these conditionals are deliberationally useful, whether or not the agent is concerned about the money at all, and whether or not they care about their neighbours' opinions. It is the agent's task, not the speaker's, to figure out whether it is more important for them that the car is reliable or whether they assign a higher utility value to the car being a status symbol. 
the conditionals that we analysed so far involve backtracking reasoning. To show that the "inferential" account scores better than the alternative, let us consider the case of a deliberationally useless conditional which does not depend on any causal consideration at all.

In Section 3, we argued that the presence of backtracking grounds is not only insufficient for a conditional to be deliberationally useless, but it is also not necessary. The sentence $\left(\mathrm{I}_{3}\right)$ :

(13) If Felix wears that jacket, he will be able to wear just about anything.

is an example of a conditional which is not assertable in the context of deliberation, although it is not based on backtracking grounds. Felix's wearing a piece of extremely extravagant clothing provides evidential support for the claim that he can wear just about anything, but there is no causal relation, forward or backtracking, between the two facts. There is, however, an abductive step involved in reasoning from the conditional's antecedent to its consequent: Felix's eccentric costume, given his aspirations to become a fashionista, can be explained by his newly gained self-confidence, which in turn allows the speaker to infer that there are hardly any limits to what Felix can wear.

It is not only the case that the "inferential" account allows us to identify deliberationally useless conditionals when DeRose's analysis fails, but it can also explain why these conditionals are deliberationally useless, that is, unassertable in contexts of deliberation. In fact, it should be hardly surprising that abductive inference, i.e. an inference to the best explanation, does not provide us with any information that we could use to make a rational decision regarding the premises of that inference. If we are trying to decide whether we should $\varphi$, what we are interested in is what $\varphi$ can bring about, not in the explanation of why we $\varphi$, be it in terms of causes, reasons, or dispositions. Someone explaining to us what our decision to do $\varphi$ would be an indication or a symptom of would not be helpful at all.

Finally, let me remark on the two different roles that the context of utterance, which may be characterised as "the concrete situation in which a conversation takes place, a situation with a more or less definite group of participants with certain beliefs, including beliefs about what the others know and believe, and certain interests and purposes, both common interests and purposes, and interests and purposes that are recognized to diverge" (Stalnaker 20I4: I4, emphasis mine), plays in the process of production and interpretation of indicative conditionals. On the one hand, context provides the hearer with the information necessary to interpret context-sensitive expressions such as indexicals or quantifiers, and, as we have argued earlier, to distinguish between different types of inferences that a conditional may express. On the other hand, a context should guide a speaker in deciding whether a particular conditional is assertable.

Recognising that a given context is someone's context of deliberation should prevent a speaker from asserting an abductive inferential conditional, even if, in a purely epistemic context, the speaker would be fully warranted in its assertion. Such a context-sensitivity is not unique to conditionals. The assertability of any given sentence does not only depend on its content and the degree to which the speaker is warranted in asserting it, but also on the context of utterance (see, e.g., DeRose 2002; Douven 2006). In other words, the assertability conditions implicitly refer to context. This is why the same sentence - be it a categorical statement or a conditional - might be assertable in one context and 
unassertable in another context. For instance, in the context in which one of the interlocutors is recognised as being hungry, the sentence:

(30) There is a bakery around the corner.

will not be assertable for a speaker who knows that the bakery is closed, since it would be interpreted as a suggestion that the hungry interlocutor could buy something to eat in that bakery (cf. Grice 1989: 32). Nevertheless, (30) would be perfectly assertable in a context in which the interlocutor is interested in renting an apartment and is asking about the facilities available in the neighbourhood. In this case, it does not matter whether or not the bakery is open at the time of the utterance, and the sentence's assertability is not affected by the speaker's knowledge about it. If a conditional of deliberation is asserted in a context of deliberation, and the hearer believes that the speaker is aware of that, they will assume that the asserted conditional is deliberationally useful and, consequently, reject the abductive inferential interpretation of that conditional, analogously to the hungry interlocutor assuming that the assertion of (30) is being made precisely because it is relevant to the problem at hand.

As we have seen, there is much more to indicative conditionals than the truth values of their clauses or the conditional probabilities of their consequents given their antecedents. There is also a greater variation of possible links between the antecedents and consequents of indicative conditionals than forward and backtracking causal relations. Taking into account that different conditionals can parallel arguments of various types seems to be the key to understanding why some perfectly justified indicatives can lead a deliberating agent astray. The "inferential" account of conditionals allows us to identify those conditionals that are deliberationally useless, but also provides a reason and an explanation for their infamous role in the process of decision making. Conditionals that are deliberationally useless are abductive inferential conditionals, or at least they significantly depend on an explanatory reasoning. The question of the nature of this dependence shall, for the moment, remain open. ${ }^{23}$

\section{REFERENCES}

Adams, E. W. 1970. 'Subjunctive and Indicative Conditionals.' Foundations of Language, 6(I): 89-94.

Bach, K. 2008. 'Applying Pragmatics to Epistemology.' Philosophical Issues, I 8: 68-88.

Bennett, J. 2003. A Philosophical Guide to Conditionals. Oxford: Oxford University Press.

Burch, R. 20I4. 'Charles Sanders Peirce.' In E. N. Zalta (ed.), Stanford Encyclopedia of Philosophy, http://plato.stanford.edu/archives/win2or 4 /entries/peirce/.

Dancygier, B. and Sweetser, E. 2005. Mental Spaces in Grammar: Conditional Constructions. Cambridge: Cambridge University Press.

Declerck, R. and Reed, S. 200I. Conditionals: A Comprehensive Empirical Analysis. Berlin: Mouton de Gruyter.

23 I am grateful to Wojciech Rostworowski and to an anonymous referee at Episteme for extremely helpful comments on the previous version of the manuscript. This research was supported by the Alexander von Humboldt Foundation and by the Senior Researcher in Residence Programme of the Center for Advanced Study, LMU Munich. 
DeRose, K. 2002. 'Assertion, Knowledge, and Context.' Philosophical Review, III(2): I67-203.

- 20I0. 'The Conditionals of Deliberation.' Mind, I I9: I-42.

Douven, I. 2006. 'Assertion, Knowledge, and Rational Credibility.' Philosophical Review, I I 5(4): 449-85.

2010. 'The Pragmatics of Belief.' Journal of Pragmatics, $42(\mathrm{I}): 35-47$.

- 2016. The Epistemology of Indicative Conditionals. Cambridge: Cambridge University Press.

- 2017. 'How to Account for the Oddness of Missing-link Conditionals.' Synthese, 194(5): I 54 I -54 .

Douven, I. and Verbrugge, S. 2010. 'The Adams Family.' Cognition, I I7: 302-I 8.

Edgington, D. I995. 'On Conditionals.' Mind, I04(414): 235-329.

Douven, I., Elqayam, S., Singmann, H. and van Wijnbergen-Huitink, J. 20I8. 'Conditionals and Inferential Connections: A Hypothetical Inferential Theory.' Cognitive Psychology, ror: 50-8 I.

Elder, C.-H. and Jaszczolt, K. M. 20I6. 'Towards a Pragmatic Category of Conditionals.' Journal of Pragmatics, 98: 36-53.

Evans, J. S. B. T. and Over, D. E. 2004. If. Oxford: Oxford University Press.

Gibbard, A. I98 I. 'Two Recent Theories of Conditionals.' In W. L. Harper, R. Stalnaker and G. Pearce (eds), Ifs: Conditionals, Belief, Decision, Chance, and Time, pp. 2 I I-247. Dordrecht: Reidel. and Harper, W. L. 1978. 'Counterfactuals and Two Kinds of Expected Utility.' In W. L. Harper, R. Stalnaker and G. Pearce (eds), Ifs: Conditionals, Belief, Decision, Chance, and Time, pp. I 53-90. Dordrecht: Reidel.

Grice, H. P. 1989. Studies in the Way of Words. Cambridge, MA: Harvard University Press.

Jeffrey, R. C. 1983 . The Logic of Decision, 2nd edn. Chicago: University of Chicago Press.

Joyce, J. M. I999. The Foundations of Causal Decision Theory. Cambridge Studies in Probability, Induction, and Decision Theory. Cambridge: Cambridge University Press.

Krzyżanowska, K., Wenmackers, S. and Douven, I. 20I3. 'Inferential Conditionals and Evidentiality.' Journal of Logic, Language and Information, 22(3): 3 I 5-34.

- 20I4. 'Rethinking Gibbard's Riverboat Argument.' Studia Logica, IO2(4): 77I-92.

Lewis, D. 1973. Counterfactuals. Oxford: Blackwell.

- 1976. 'Probabilities of Conditionals and Conditional Probabilities.' Philosophical Review, $85(3): 297-3$ I 5 .

— I98I. 'Causal Decision Theory.' Australasian Journal of Philosophy, 59(I): 5-30.

Mackie, J. L. 1973. Truth, Probability and Paradox: Studies in Philosophical Logic. Oxford: Clarendon Press.

Mill, J. S. I 843. A System of Logic, Ratiocinative and Inductive: Being A Connected View of the Principles of Evidence and the Methods of Scientific Investigation, Vol. I. London: John W. Parker.

Nozick, R. I969. 'Newcomb's Problem and Two Principles of Choice.' In N. Rescher (ed.), Essays in Honor of Carl G. Hempel, pp. I I4-46. Dordrecht: D. Reidel.

Ramsey, F. P. 1929/1990. 'General Propositions and Causality.' In D. H. Mellor (ed.), Philosophical Papers, pp. $145-63$. Cambridge: Cambridge University Press.

Sanford, D. H. 1989. If P, Then Q: Conditionals and the Foundations of Reasoning. London: Routledge.

Skovgaard-Olsen, N. 20I4. Making Ranking Theory Useful for Psychology of Reasoning. PhD thesis, Universität Konstanz.

Skovgaard-Olsen, N., Singmann, H. and Klauer, K. C. 20I6. 'The Relevance Effect and Conditionals.' Cognition, I 50: 26-36.

Spohn, W. 2013. 'A Ranking-theoretic Approach to Conditionals.' Cognitive Science, 37: 1074-Io6.

Stalnaker, R. 20I4. Context. Oxford: Oxford University Press.

Stalnaker, R. C. I968. 'A Theory of Conditionals.' In N. Rescher (ed.), Studies in Logical Theory. American Philosophical Quarterly Monograph Series, Vol. 2, pp. 98-I I 2. Oxford: Blackwell. 1975. 'Indicative Conditionals.' Philosophia, 5(3): 269-86.

Sweetser, E. 1990. From Etymology to Pragmatics: Metaphorical and Cultural Aspects of Semantic Structures. Cambridge: Cambridge University Press.

Verbrugge, S. 2007. A Psycholinguistic Analysis of Inferential Conditional Sentences. $\mathrm{PhD}$ thesis, Katholieke Universiteit Leuven. 
_ Dieussaert, K., Schaeken, W., Smessaert, H. and Belle, W. V. 2007. 'Pronounced Inferences: A Study on Inferential Conditionals.' Thinking and Reasoning, I3 (2): 105-33.

Williamson, T. 2000. Knowledge and Its Limits. Oxford: Oxford University Press.

KAROLINA KRZYŻANOWSKA is a postdoctoral researcher at the Institute for Logic, Language and Computation of the University of Amsterdam. She obtained her PhD in Philosophy from the University of Groningen in 2015. Before coming to Amsterdam, she was a research fellow at the Munich Center for Mathematical Philosophy and at the Center for Advanced Studies of the LMU Munich. Her research interests include semantics and pragmatics of conditionals, and their role in reasoning and decision making. 